\title{
Unique crystal morphologies of glycine grown from octanoic acid-in-water emulsions.
}

\author{
Catherine E. Nicholson, Sharon J. Cooper*, Matthew J. Jamieson \\ Department of Chemistry, University of Durham, South Road, Durham. DH1 3LE
}

\section{Supporting Information}

\section{Materials and Preparation Methods}

Glycine, sodium chloride, octanoic acid, decane, acetic acid, Tween 20, Span 20 and Brij 30 were obtained from Aldrich and used without further purification. Ultrapure water with a resistivity of $18 \mathrm{M} \Omega \mathrm{cm}$ was provided by a UHQ water filtration unit. The $21.5 \mathrm{wt} \%$ glycine solutions were prepared by mixing $21.5 \mathrm{~g}$ of glycine and $78.5 \mathrm{~g}$ of UHQ water and then treating in a hot $\left(\sim 60^{\circ} \mathrm{C}\right)$ ultrasonic bath for at least 2 hours. These glycine solutions were saturated at $28.1{ }^{\circ} \mathrm{C}$ (glycine solubility is calculated from: $\mathrm{S}=13.63+0.49 \mathrm{~T}$, where $\mathrm{S}$ is the solubility of glycine in $100 \mathrm{~g}$ of water and $\mathrm{T}$ is the temperature in degrees Celsius), and so were stored at $40{ }^{\circ} \mathrm{C}$ until their subsequent use within a few days. Emulsions were prepared as $2 \mathrm{~g}$ aliquots with an octanoic acid weight fraction of $60 \%$. The surfactant blends typically consisted of $8: 1$ Tween 20:Brij 30 or 8:1 Span 20:Brij 30. The total surfactant concentration in each emulsion was $9 \mathrm{wt} \%$ for preparation of the macroporous glycine crystals, and $50 \mathrm{wt} \%$ for growth of the complex dendritic forms. The emulsion preparation method was as follows. The required amounts of octanoic acid and surfactants were mixed by vortexing for $10 \mathrm{~s}$ at $2500 \mathrm{rpm}$, and the solution heated to $40{ }^{\circ} \mathrm{C}$. The glycine solution was then added and the emulsion obtained by vortexing for 1 minute at $2500 \mathrm{rpm}$. For the preliminary experiments on sodium chloride crystallization from octanoic acid emulsions, we used $19.4 \mathrm{wt} \%$ of sodium chloride in the aqueous solutions, an octanoic acid weight fraction of $60 \%$ and 9 wt $\%$ of surfactant comprising 8:1 Tween 20:Brij 30. The solution and emulsion preparation methods were the same as for the glycine case.

\section{Optical Microscopy Studies}

Optical microscopy data were acquired on an Olympus BX50 microscope equipped with a digital camera and Linksys 32 software to enable image capture and processing. 1 drop $(\sim 10 \mathrm{mg})$ of the freshly prepared emulsion was placed on a glass coverslip on the Linkam heating/cooling block, which had a central hole (1 $\mathrm{mm}$ by $4 \mathrm{~mm}$ ), to allow light transmission through the sample. The block was cooled by liquid nitrogen and the resulting nitrogen gas was used to purge the sample environment. Glycine crystallization was induced by cooling from $25^{\circ} \mathrm{C}$ to $20^{\circ} \mathrm{C}$ at $1{ }^{\circ} \mathrm{C}$ minute ${ }^{-1}$. Sodium chloride crystallization was induced on water evaporation at ambient temperature.

\section{ESEM Studies}

Environmental scanning electron microscopy (ESEM) studies were conducted using a Philips XL30 ESEM operating in low vacuum mode with a water vapour pressure of 0.4 Torr. An electron backscatter detector was used to enable visualization of the samples.

\section{X-ray Diffraction Studies}

X-ray diffraction studies were undertaken using copper $K \alpha$ radiation $(\lambda=1.5418 \AA$ ) from a sealed tube source on a Bruker D8 system incorporating a horizontal sample stage and two- 
dimensional multi-wire detector. The X-ray incidence angle was varied from $8^{\circ}$ to $12^{\circ}$. Polymorph determination was performed in situ, and also at later times after all the glycine had crystallized following the room temperature evaporation of all the water. Characteristic diffraction signals for the $\alpha$-, $\beta$ - and $\gamma$-polymorphs of glycine are given in Table S1, together with the unit cell data for each polymorph.

\begin{tabular}{|l|c|c|c|}
\hline & \multicolumn{3}{|c|}{ Polymorphic Form } \\
\hline & $\alpha$ & $\beta$ & $\gamma$ \\
\hline Unit cell parameters $^{1}$ & $\begin{array}{c}\mathrm{a}=5.11 \AA, \mathrm{b}=12.04 \AA, \\
\mathrm{c}=5.46 \AA, \beta=111.82^{\circ}\end{array}$ & $\begin{array}{c}\mathrm{a}=5.09 \AA, \mathrm{b}=6.29 \AA, \\
\mathrm{c}=5.38 \AA, \beta=113.21^{\circ}\end{array}$ & $\begin{array}{c}\mathrm{a}=7.04 \AA, \mathrm{b}=7.04 \AA, \\
\mathrm{c}=5.48 \AA, \gamma=120^{\circ}\end{array}$ \\
\hline $\begin{array}{l}\text { Characteristic } 2 \theta \\
\text { values for copper } \\
\text { K } \alpha \text { X-ray radiation }\end{array}$ & $\begin{array}{c}19.0^{\circ}(011), 23.9^{\circ}(120), \\
29.7^{\circ}(040)\end{array}$ & $\begin{array}{c}17.9^{\circ}(001), 23.8^{\circ}(110), \\
28.5^{\circ}(020)\end{array}$ & $\begin{array}{c}14.5^{\circ}(100), 21.8^{\circ} \\
(101), 25.3^{\circ}(110)\end{array}$ \\
\hline
\end{tabular}

Table S1. Characteristic X-ray diffraction peaks and unit cell data for polymorphs of glycine.

\section{Crystal Morphologies}

Typical examples of the glycine macroporous and fenestrated intergrown crystals are shown in Figure S1, whilst typical examples of the glycine dendritic forms are shown in Figure S2.

Representative optical micrographs from the preliminary experiments on sodium chloride crystallization from octanoic acid emulsions are compared with the typical aqueous phase morphology in Figure S3. Two distinct morphologies for the sodium chloride crystals were obtained. In particular, the first crystals that grew developed into highly pitted and fragmental cubic crystals, in which there was evidence of complete droplet inclusion (see Figures S3b and S3c). At later stages, crystal dendrites were formed due to partial droplet encapsulation (see Figure S3d). Hence, it is apparent that octanoic acid droplets can also adhere onto these sodium chloride crystals to produce anomalous crystal morphologies, despite the stronger ionic bonding in this system.

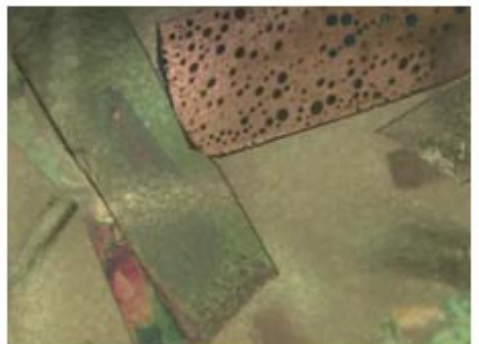

a)

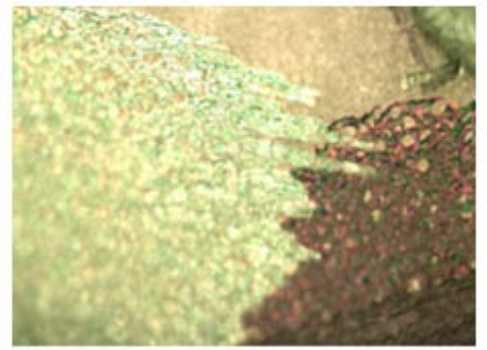

b)
$0.5 \mathrm{~mm}$

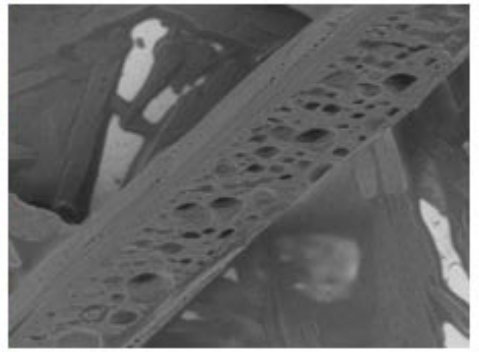

c)
$0.25 \mathrm{~mm}$

Figure S1. a) and b) Optical micrographs under crossed-polarizers showing macroporous morphologies of $\beta$ glycine crystals grown from octanoic acid emulsions. Note that a red tint plate has been used to aid crystal distinction. The crystals shown in a) and b) grew from 9 wt\% surfactant solutions comprising 8:1 Span 20:Brij30 and 8:1 Tween 20:Brij 30, respectively, to produce the macroporous single crystals and the fenestrated morphology arising from intergrown co-aligned dendrite branches. c) ESEM micrograph of macroporous glycine crystals extracted from an octanoic acid emulsion stabilized with 9 wt\% of 8:1 Span 20:Brij 30. 


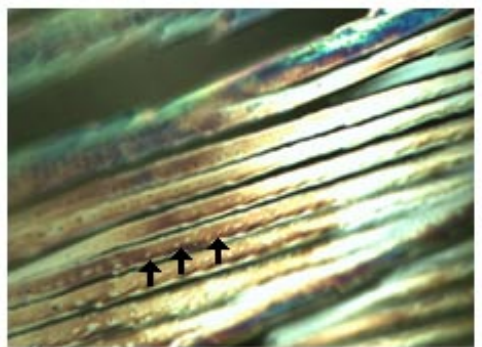

a)

$0.1 \mathrm{~mm}$

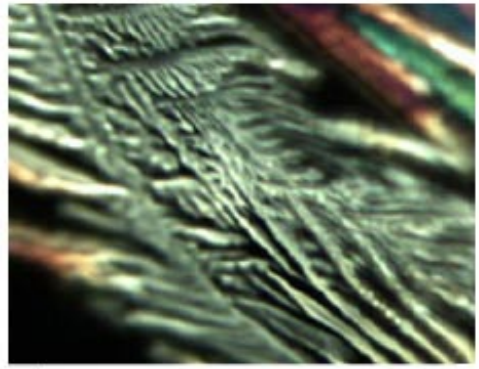

c)

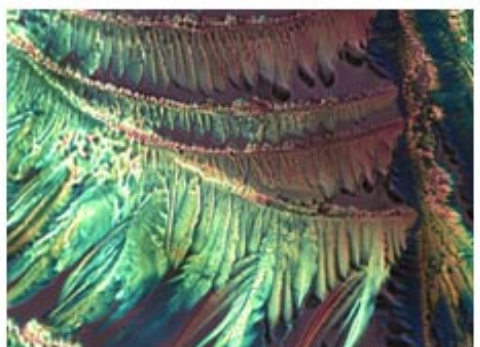

b) $0.1 \mathrm{~mm}$

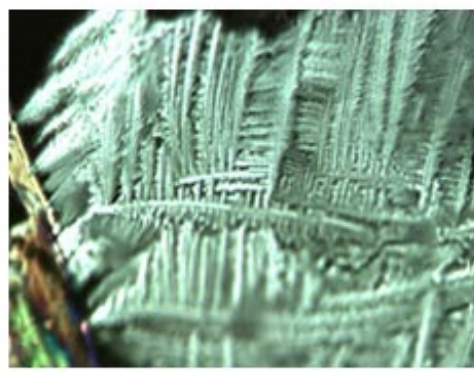

d)

$0.05 \mathrm{~mm}$

Figure S2. Optical micrographs under crossed-polarizers showing the dendritic morphologies of $\beta$-glycine crystals grown from octanoic acid emulsions comprising 50 wt\% surfactants solutions with 8:1 Span 20:Brij

30. a) Co-aligned dendrites. The arrows indicate some of the regions in which adhered droplets can be seen. b), c) and d) complex dendritic morphologies. b) shows an earlier crystallization stage than c) and d) and hence the dendritic branches are less intergrown. Note a red tint plate has been used in images $a$ ) and $b$ ) to aid crystal distinction

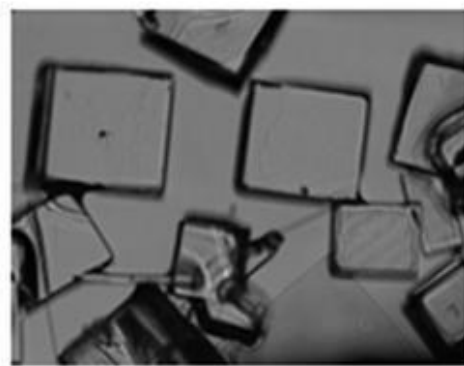

a)

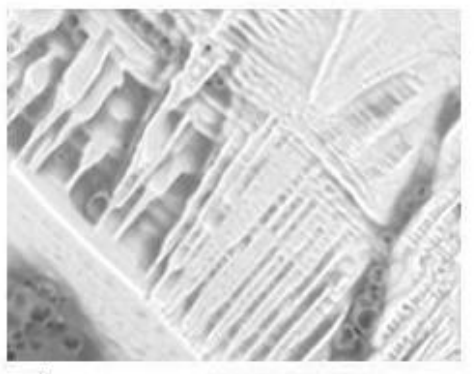

c)
$0.02 \mathrm{~mm}$

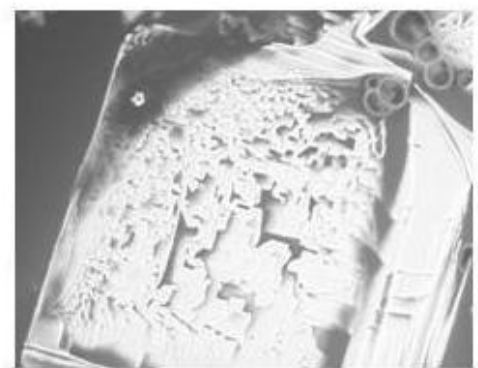

b)

$0.05 \mathrm{~mm}$

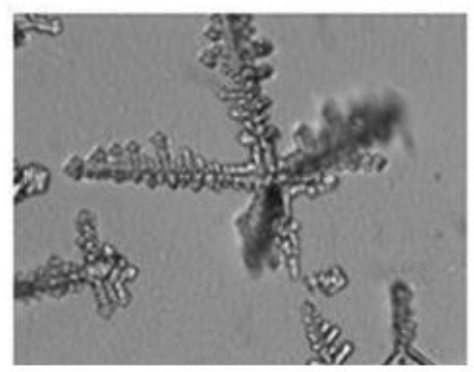

d)

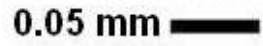

Figure S3. a) Optical micrograph under crossed-polarizers showing the normal aqueous phase crystal morphology of sodium chloride. b) and c) ESEM micrographs showing the crystal morphology that develops during initial crystallization from octanoic acid emulsions. The crystals have a pitted and fragmental appearance due to droplet adhesion. Full droplet encapsulation is also apparent in a few areas.

d) Optical micrograph under crossed-polarizers showing the dendritic morphology that develops due to partial droplet encapsulation during late-stage crystallization from octanoic acid emulsions. 
The movies included in the Supporting Information show crystallization of the macroporous glycine crystals from a 9 wt\% 8:1 Span 20:Brij 30 emulsion, see file entitled "SJC Supporting Info Macroporous Glycine Movie", and crystallization from the $50 \mathrm{wt} \%$ surfactant solutions comprising 8:1 Span 20:Brij 30 to produce the glycine dendritic forms, see file entitled "SJC Supporting Info Dendritic Glycine Movie". The micrograph length represents $0.25 \mathrm{~mm}$ in both movies. In the movie showing the macroporous glycine morphology, an image was captured every 3 seconds, with 160 images making up the movie. In the movie showing the dendritic morphology, an image was captured every second, with 53 images contained in the movie.

\section{X-ray Diffraction Patterns}

The nonionic surfactant blends promoted the formation of the $\beta$-polymorph of glycine, as shown by the presence of the strong near-meridional 001 diffraction signal at $2 \theta=17.9^{\circ}$, see Figure S4a. This diffraction signal dominates the diffraction spectra, as the crystals grow as thin plates or dendrites with the 001 plane typically lying horizontal, and hence the majority of crystals bathed in X-rays can potentially contribute to this diffraction peak for an X-ray incident angle of $\sim 9^{\circ}$. Figure S4a also shows the $11 \overline{2}$ signal at $2 \theta=36.7^{\circ}$, which could also be observed on appropriately oriented samples. The diffraction patterns from the macroporous crystals showed isolated discrete spots, whereas those from the coaligned dendrites and complex dendritic morphologies often showed a series of closely spaced spots, as shown in Figure S4a, due to dendrite divergence.
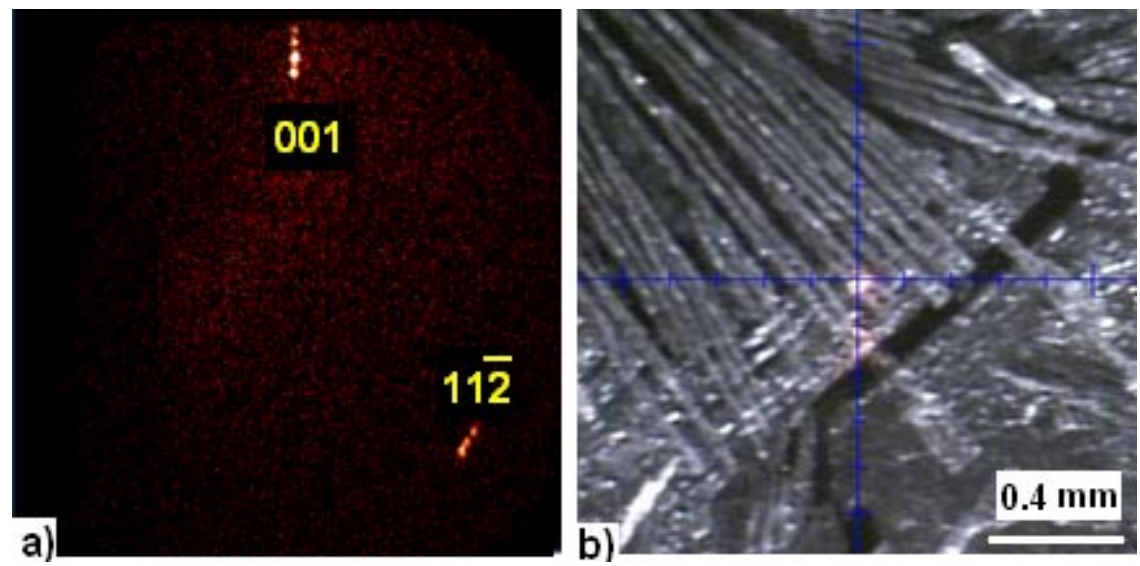

Figure S4. a) 2-dimensional X-ray diffraction patterns showing 001 and $11 \overline{2}$ diffraction peaks at $2 \theta=17.9^{\circ}$ and $36.7^{\circ}$, respectively, due to $\beta$-phase crystallization of glycine dendrites from octanoic acid-in-water emulsions containing $50 \mathrm{wt} \%$ surfactant. Note the appearance of several closely spaced diffraction spots, rather than single discrete spots due to dendrite divergence. $b$ ) Optical micrograph of the dendritic sample from which the diffraction pattern was obtained. The region at the centre of the cross-hairs shows the region probed by the X-ray beam.

\section{Reference}

1. Perlovich, G. L.; Hansen, L. K.; Bauer-Brandl, A. J. Therm. Anal. Cal. 2001, 66, 699715. 